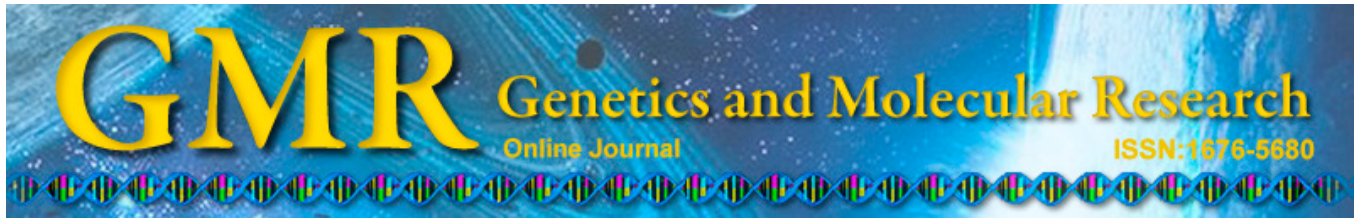

\title{
Selected representative microsatellite loci for genetic monitoring and population structure analysis of miniature swine
}

\author{
C. Wang ${ }^{1,2 *}$, L.L. Xu ${ }^{1 *}$, Y.H. Wu ${ }^{1}$, X.Y. Du ${ }^{1}$, X.Y. Huo ${ }^{1}$, J. Gao ${ }^{1}$, Z.K. Li ${ }^{1}$, \\ Y.C. Li ${ }^{1}$ and Z.W. Chen ${ }^{1}$ \\ ${ }^{1}$ Department of Laboratory Animal Science, School of Basic Medical Sciences, \\ Capital Medical University, Beijing, China \\ ${ }^{2}$ Department of Animal Physiology, College of Biological Sciences, \\ China Agricultural University, Beijing, China \\ *These authors contributed equally to this study. \\ Corresponding author: Z.W. Chen \\ E-mail: czwen@ccmu.edu.cn
}

Genet. Mol. Res. 14 (2): 3910-3920 (2015)

Received April 1, 2014

Accepted September 11, 2014

Published April 27, 2015

DOI http://dx.doi.org/10.4238/2015.April.27.5

\begin{abstract}
To optimize the combination of microsatellite loci for genetic control of outbred swine stocks, 32 of 100 loci distributed among almost all chromosomes (except 12) were screened out by $1.5 \%$ agarose, $8 \%$ polyacrylamide gel and capillary electrophoresis scanning among 3 miniature swine outbred stocks, namely Bama (BM), Guizhou (GZ) and Tibeta (TB). The mean total and effective allele numbers among these stocks were 12.1 and 5.9, respectively. The mean heterozygosity for these breeds was $0.5428,0.6978$ and 0.7646 , and polymorphism information content was $0.5469,0.7296$ and 0.7663 , respectively. Accordingly, hereditary variation from low to high was $\mathrm{BM}<\mathrm{GZ}<\mathrm{TB}$. This showed that the genetic relationship between BM and GZ pigs was closer, and both were distant from TB. Additionally, the effectiveness of the 32-locus combination for evaluation of genetic quality was demonstrated in Changchun-junmo-1 (CJ-1), a standard
\end{abstract}


outbred Chinese pig stock, in which the mean total and effective allele numbers and mean heterozygosity were $6.1613,3.8483$ and 0.6903 , respectively. Since our results were consistent with the breeding pedigrees, the 32 loci could be used for both genetic monitoring within the individual outbred miniature swine stocks and population structure analysis between them.

Key words: Genetic monitor; Microsatellite DNA; Miniature swine; Population genetic structure

\section{INTRODUCTION}

Pigs are important for biomedical research due to their biochemical, anatomical and physiological similarities with humans (Mortensen et al., 1998; Droc et al., 2009), and their ideal sizes, high reproduction and easy management (Bode, 2010; Ross et al., 2012). Chinese outbred stocks of miniature swine, including Wuzhishan (WZS), Bama (BM), Guizhou (GZ), and Tibeta (TB), have been widely used for decades (Esteban et al., 2009; Sun et al., 2011; Cao et al., 2012). However, because the genetic quality standard has not been established, it is difficult to monitor the genetic quality within each stock and/or unify the quality among stocks, which becomes one of the obstacles for its wide use.

As a complementary strategy to traditional phenotype driven genetic monitoring, the study of molecular genetic structure and diversity of various swine stocks is useful (Wilkinson et al., 2011). Microsatellites are useful for analysis of genetic traits, population structures and relationships between domestic (Fredholm et al., 1993) and Chinese miniature swine (Yang et al., 2003; Kim et al., 2005; Megens et al., 2008). The microsatellite loci that have been used in miniature swine are either from organizations (Kim et al., 2005; Yao et al., 2006) or selected by researchers (Megens et al., 2008). Unfortunately, some of the loci have poor polymorphic characters, and the number of effective alleles and size of the same locus vary (Yao et al., 2006; Fang et al., 2009; Chang et al., 2009). As a result, comparison of the molecular genetic traits of stocks based on these studies is not possible. The objective of this study was to screen a combination of microsatellite loci for studying the genetic structure of Chinese outbred swine stocks and to monitor their quality.

\section{MATERIAL AND METHODS}

\section{Animals and tissue sampling}

Three outbred stocks of miniature swine on normal diet and management, namely $\mathrm{BM}, \mathrm{GZ}$ and TB, together with Changchun-junmo-1(CJ-1), which has been bred for more than 20 generations and recognized as a standard outbred Chinese pig stock (Sun et al., 2000), were used in this study. These pigs were raised by different indigenous conservation breeding farms or institutions. All of them had a recorded pedigree. The general information of these stocks is presented in Table 1. All of the experiments and procedures performed on the animals were approved by the animal welfare committee of Capital Medical University, Beijing, China. Based on the numbers of each pig population, 35 pigs were chosen for each miniature swine stock for sample collection, while 40 pigs were used for CJ-1. Animal selection in each 
breed was generally based on random selection, nearly half sires and half dams. To enlarge the sampling coverage ratio and obtain representative results, only one piglet was picked out from a mother. Before sampling, each pig was safely restrained and the right ear was sterilized. The ear tissue with a size of about $2.0 \times 0.5 \mathrm{~cm}$ was quickly cut out from the outer edge of the ear with an ear clamp. The sample for each pig was quickly put into a sterile tube and stored at $-80^{\circ} \mathrm{C}$ for later use.

\begin{tabular}{|c|c|c|c|c|}
\hline Breeding started & Origin (Province) & Conservational institutions & Generations & Samples \\
\hline BM, 1987 & Guang Xi & Guang Xi University & $>20$ & 35 \\
\hline GZ, 1982 & Gui Zhou & GCTCM & $>15$ & 35 \\
\hline TB, 2004 & Tibet & SMU-SBFG & 4 & 35 \\
\hline CJ-1, 1988 & Ji Lin & Ji Lin University & $>20$ & 40 \\
\hline
\end{tabular}

GCTCM means Guizhou College of Traditional Chinese Medicine. SMU-SBFG means Southern Medical University \& Shenzhen Bright Farm Group.

\section{Candidate microsatellite resource}

The microsatellite resource was composed of 100 loci, including 27 loci recommended by FAO (Food and Agriculture Organization of the United Nations) and ISAG (International Society of Animal Genetics) (http://www.isag.us), and 21 demonstrated loci of Chinese miniature swine (Li et al., 2004); the other loci came from general reports (Rohre et al., 1994) and GenBank (Table S1). The criteria for selecting these loci were having satisfactory polymorphisms and covering all 19 chromosomes except the Y chromosome. Primers of these loci were synthesized by Sangon Biotech (Shanghai) Co., Ltd. (Shanghai, China). All of the chemicals and reagents used in microsatellite proliferation and detection were purchased from Sigma-Aldrich Inc. (St. Louis, MO, USA) unless otherwise indicated.

\section{DNA extraction}

Total genomic DNA of each sample was extracted from frozen ear tissue. DNA was isolated by phenol-chloroform extraction and ethanol precipitation according to previously described methods (Zuo et al., 2012). The DNAs extracted were stored in TE buffer at $4^{\circ} \mathrm{C}$. The DNA samples were then quantitated by measuring the A260/A280 value with a microplate absorbance reader (Bio-Rad 680, USA), and the quality of these samples was further evaluated by $0.8 \%$ agarose gel electrophoresis. DNA samples were diluted to $100 \mathrm{ng} / \mu \mathrm{L}$ and stored at $-20^{\circ} \mathrm{C}$ for later use as templates in polymerase chain reactions (PCRs).

\section{PCR procedure}

Microsatellites were amplified by PCR. To minimize the variations that could exist among individual pigs, and to amplify as many polymorphic alleles as possible for each microsatellite locus, every 9 DNA samples that were evenly coming from the three stocks were mixed. In brief, for each stock, three samples were randomly picked out and $20 \mu \mathrm{L}$ DNA solution $(100 \mathrm{ng} / \mu \mathrm{L})$ from each sample were gently mixed. The mixture was used as the template for DNA amplification. In brief, each $15-\mu \mathrm{L}$ PCR amplification system generally contained 
the following reagents: $1.5 \mu \mathrm{L} 10 \mathrm{X}$ buffer, $1.0 \mu \mathrm{L} 1.0 \mu \mathrm{M}$ of each primer, $1.0 \mu \mathrm{L} 100 \mu \mathrm{M}$ x 4 dNTPs (Takara Co. Ltd., Tokyo, Japan), $1.0 \mu \mathrm{L} 1.0 \mathrm{U}$ Taq high-fidelity DNA polymerase (Takara), and $1.0 \mu \mathrm{L}(100 \mathrm{ng})$ template DNA. PCR amplifications were performed in a gradient thermal cycler (Model:ALS1296, Bio-Rad Inc., Watertown, MA, USA) using the following protocol: predenaturation at $94^{\circ} \mathrm{C}$ for $5 \mathrm{~min}$; 35 cycles of denaturation at $94^{\circ} \mathrm{C}$ for $30 \mathrm{~s}$, annealing for $30 \mathrm{~s}$, and extension at $72^{\circ} \mathrm{C}$ for $30 \mathrm{~s}$; and a final extension at $72^{\circ} \mathrm{C}$ for $7 \mathrm{~min}$. The products were stored at $4^{\circ} \mathrm{C}$ for further microsatellite analysis.

\section{Stepwise microsatellite analysis}

At stage one, agarose electrophoresis based screening. For each microsatellite amplification reaction, PCR was performed in two steps. In the first step, the PCR procedure was faithfully performed according to the originally reported parameters, including the concentration of $\mathrm{MgCl}_{2}$ and the annealing temperature. An 8.0- $\mu \mathrm{L}$ volume of each product was stained with ethidium bromide, and the bands of each product were separated by $1.5 \%$ agarose electrophoresis at $140 \mathrm{~V}$ for $30 \mathrm{~min}$ and visualized with a UV transilluminator (VilBerLouRMAT Inc.). Secondly, any PCR producing unclear bands was optimized by modulating the concentration of $\mathrm{MgCl}_{2}$ (Takara) from 1.5-3.0 mM, and by choosing the optimal annealing temperature point by comparing the quality of PCR products at seven gradually varied temperature points around the reported point. Those loci that had more than three alleles within the reported product size ranges were chose for later selections. The experiments were repeated three times for each microsatellite.

At stage two, polyacrylamide gel electrophoresis-based screening. The microsatellite loci selected from stage one were further examined by denaturing $8 \%$ polyacrylamide sequencing gels at $3 \mathrm{~V} / \mathrm{cm}$ for $1 \mathrm{~h}$, which were then stained in a $\mathrm{AgNO}_{3}$ solution and visualized with a UV transilluminator (VilBerLouRMAT Inc.). At this stage, those loci that had more than 5 alleles/each locus were screened out for the next selection step. The experiments were repeated three times for each microsatellite.

At stage three, capillary electrophoresis-based screening. A total of 105 DNA samples from the three miniature swine stocks were used for PCR amplification with fluorescently labeled primers [fluorescent dyes were 6-FAM (blue), HEX (green) and TAMRA (yellow); the internal size standard was ROX (red)]. The procedure was performed according to a previous report (Zuo et al., 2012). In brief, primers were respectively tagged at the 5 ' end with dyes first. After PCR was performed in a $15-\mu \mathrm{L}$ reaction mixture, the PCR products were mixed at a ratio of 1:3:5 (FAM:HEX:TAMRA). A 1- $\mu \mathrm{L}$ aliquot of each mixture was combined with 25 $\mu \mathrm{L}$ formamide, vortexed and then sized by capillary electrophoresis on an ABI-3730XL DNA Analyzer (PE Biosystems, USA). Of all loci screened, the loci that had more than five effective alleles were confirmed to be the representative loci for genetic monitoring and population studies. The experiments were repeated three times for each microsatellite locus.

\section{Population genetic structure analysis}

After the genotypes of each locus in every stock were systematically recorded, the genetic structures within each outbred stock were analyzed on the basis of the screened microsatellite combination. The general genetic structure indices, including the observed and effective numbers of alleles, Shannon information index, observed and effective heterozygosities, 
and average polymorphism information content (PIC), were calculated for genetic variation analysis within the stocks. In addition, Nei's standard genetic distance between the three populations was measured as well (Nei, 1995).

\section{Applicability of the screened microsatellite locus combination in CJ-1}

The selected combination of microsatellite markers was further tested for its applicability in monitoring pig genetic background besides the miniature swine stocks. The genetic structure indices of CJ-1, such as the effective allele number, effective heterozygosity and Shannon information index were studied by amplification of the loci selected. The experiments were repeated three times for each microsatellite locus.

\section{Statistical analysis}

The genotypes of the microsatellite loci were analyzed by the GeneScan 3.7 software (Applied Biosystems). The number of alleles and the mean heterozygosity within each stock was analyzed by the GENEPOP software (Version 3.3) (May et al., 1995). The genetic distance between the three populations was measured according to Nei's standard distance method. An unweighted pair group method with arithmetic mean (UPGMA) (Sneath and Sokal, 1973) was used to construct the phylogenetic tree based on Nei's standard genetic distance.

\section{RESULTS}

\section{Miniature pig microsatellite loci selected}

To find representative microsatellite loci that could reflect the general background of each stock, a narrowed down selection procedure was performed by carefully examining the PCR products at each selection stage. After the first round of selection, 85 of 100 loci that produced one or two clear bands on $1.5 \%$ agarose gel electrophoresis for each locus were selected, and 64 of these loci had more than three polymorphic alleles among the three stocks. The selected 64 loci were then reexamined by $8.0 \%$ polyacrylamide gel electrophoresis (PAGE) . By carefully observing the bands at each locus among the three stocks, 43 microsatellite loci with more than five alleles were screened out for the third stage of selection. With the help of capillary electrophoresis scanning, 32 microsatellite loci having more than 12 identical polymorphic alleles and distributed among almost all euchromosomes (except chromosome 12) and X chromosome were finally screened out (Figure S1). The detailed optimization parameters for PCR of the loci selected and the mean effective alleles for each stock are shown in Tables 2 and 3.

\section{Genetic structures of the three stocks}

Based on analyzing the PCR results of the 32 microsatellite in 105 miniature pigs derived from the three stocks, a general statistical analysis of the genetic structures within and between the three stocks was performed. As shown in Table 4, the respective mean heterozygosity of the stocks BM, GZ and TB was $0.5428,0.6978$ and 0.7646 , which im- 
plied that genetic diversity was commonly present in each stock. The PIC values of the three groups were $0.5469,0.7296$ and 0.7663 , respectively, indicating that there was high microsatellite locus polymorphism in each stock as well. The degree of hereditary variation between the three stocks from low to high was $\mathrm{BM}<\mathrm{GZ}<\mathrm{TB}$. In addition, the genetic relationship between BM and GZ pigs was closer, whereas both of them were distant from the TB stock, according to Nei's standard distance study (Table 5).

Table 2. Optimized PCR conditions (the primer sequences, the concentration of $\mathrm{MgCl}_{2}$ and the annealing temperature), the allele numbers and the size ranges of the 32 microsatellites loci.

\begin{tabular}{|c|c|c|c|c|c|}
\hline Loci & Chromosome & $\mathrm{MgCl}_{2}(\mathrm{mM})$ & Temp. $\left({ }^{\circ} \mathrm{C}\right)$ & Allele number & Allele size range \\
\hline s0008 & 1 & 2.0 & 60 & 10 & $179-200$ \\
\hline SW974 & 1 & 2.0 & 58 & 17 & $129-175$ \\
\hline SW1092 & 1 & 2.0 & 58 & 16 & $222-308$ \\
\hline S0091 & 2 & 1.5 & 55 & 14 & $96-174$ \\
\hline SW240 & 2 & 1.5 & 58 & 11 & $92-114$ \\
\hline SW1066 & 3 & 2.0 & 60 & 19 & $166-214$ \\
\hline SW1089 & 4 & 1.5 & 58 & 10 & $142-190$ \\
\hline S0005 & 5 & 2.0 & 54 & 11 & 204-244 \\
\hline S0087 & 6 & 1.5 & 58 & 10 & $164-212$ \\
\hline SW1057 & 6 & 2.0 & 58 & 14 & $142-191$ \\
\hline SW632 & 7 & 2.0 & 54 & 9 & $148-173$ \\
\hline S0225 & 8 & 1.5 & 56 & 9 & $169-190$ \\
\hline OPN & 8 & 2.0 & 59 & 12 & $138-170$ \\
\hline SW29 & 8 & 2.0 & 61 & 12 & $133-187$ \\
\hline SW911 & 9 & 2.0 & 60 & 14 & $151-178$ \\
\hline SW511 & 9 & 1.5 & 62 & 12 & $161-196$ \\
\hline SWr158 & 10 & 2.0 & 60 & 18 & $158-200$ \\
\hline SW951 & 10 & 1.5 & 58 & 14 & $108-142$ \\
\hline SW271 & 11 & 1.5 & 58 & 13 & $111-144$ \\
\hline S0386 & 11 & 2.0 & 48 & 12 & $155-178$ \\
\hline S0068 & 13 & 2.0 & 62 & 10 & $210-256$ \\
\hline SWr1008 & 13 & 2.0 & 62 & 16 & $198-256$ \\
\hline S0007 & 14 & 2.0 & 54 & 15 & $142-192$ \\
\hline SW857 & 14 & 2.0 & 58 & 16 & $129-173$ \\
\hline SW886 & 14 & 2.0 & 58 & 12 & $144-178$ \\
\hline SW919 & 15 & 1.5 & 56 & 9 & $106-136$ \\
\hline SWr312 & 15 & 1.5 & 64 & 11 & $116-136$ \\
\hline SW81 & 16 & 1.5 & 60 & 8 & $128-144$ \\
\hline SWr1120 & 17 & 1.5 & 60 & 11 & $147-178$ \\
\hline SW787 & 18 & 1.5 & 60 & 9 & $142-164$ \\
\hline S0062 & 18 & 2.0 & 56 & 12 & $144-204$ \\
\hline S0218 & $\mathrm{X}$ & 1.5 & 54 & 11 & $158-196$ \\
\hline
\end{tabular}

There were no microsatellite loci located on the euchromosome 12 due to the bad PCR output and the poor polymorphic characters.

\section{Genetic structure of CJ-1}

To prove that the 32-locus combination could be used for reliably monitoring the genetic background characters of other Chinese outbred swine stocks besides miniature stocks, the genetic structure of CJ-1 population was examined by the microsatellite locus composition. The results showed that the mean effective allele number was 3.8483 and mean heterozygosity 0.6903 (Table 6), which perfectly matched the recorded pedigree of the stock. In addition, a PIC of 0.6761 indicated that CJ-1 was a well-established outbred stock. 
Table 3. The respective/mean allele numbers of the breeds at 32 microsatellite loci.

\begin{tabular}{|c|c|c|c|c|c|c|c|c|}
\hline \multirow{2}{*}{$\begin{array}{l}\text { Population } \\
\text { loci }\end{array}$} & \multicolumn{2}{|c|}{ Mean data } & \multicolumn{2}{|c|}{$\mathrm{BM}$} & \multicolumn{2}{|c|}{ GZ } & \multicolumn{2}{|c|}{ TB } \\
\hline & OAN & EAN & OAN & EAN & OAN & EAN & OAN & EAN \\
\hline S0008 & 10.0000 & 5.3438 & 5.0000 & 1.6655 & 8.0000 & 4.1965 & 9.0000 & 6.5597 \\
\hline Sw974 & 16.0000 & 6.5973 & 4.0000 & 1.5538 & 8.0000 & 3.3256 & 10.0000 & 7.6880 \\
\hline Sw1092 & 16.0000 & 5.2071 & 10.0000 & 3.7033 & 8.0000 & 3.3660 & 7.0000 & 3.5993 \\
\hline S0091 & 14.0000 & 7.2681 & 5.0000 & 2.4484 & 5.0000 & 4.5330 & 7.0000 & 3.7393 \\
\hline Sw240 & 11.0000 & 5.1698 & 3.0000 & 1.4311 & 8.0000 & 4.1602 & 9.0000 & 4.9409 \\
\hline Sw1066 & 19.0000 & 4.5424 & 5.0000 & 1.7100 & 9.0000 & 2.8182 & 11.0000 & 5.6364 \\
\hline Sw1089 & 10.0000 & 4.8077 & 3.0000 & 2.0512 & 5.0000 & 3.1457 & 7.0000 & 2.9165 \\
\hline S0005 & 11.0000 & 5.8598 & 6.0000 & 2.1644 & 5.0000 & 2.2746 & 8.0000 & 3.8828 \\
\hline S0087 & 10.0000 & 4.2129 & 6.0000 & 3.0950 & 7.0000 & 3.1508 & 7.0000 & 1.6905 \\
\hline Sw1057 & 14.0000 & 8.0046 & 7.0000 & 3.2632 & 8.0000 & 4.4083 & 7.0000 & 3.3601 \\
\hline Sw632 & 9.0000 & 5.0417 & 4.0000 & 2.2691 & 6.0000 & 5.5549 & 9.0000 & 3.8517 \\
\hline S0225 & 9.0000 & 6.4425 & 4.0000 & 2.0168 & 7.0000 & 5.5389 & 7.0000 & 5.2948 \\
\hline Opn & 12.0000 & 8.2845 & 6.0000 & 2.3297 & 7.0000 & 4.5653 & 9.0000 & 6.4497 \\
\hline Sw29 & 12.0000 & 5.3670 & 3.0000 & 1.1027 & 7.0000 & 2.6293 & 7.0000 & 4.3094 \\
\hline Sw911 & 14.0000 & 4.6428 & 5.0000 & 1.2653 & 8.0000 & 5.3687 & 9.0000 & 4.0894 \\
\hline Sw511 & 12.0000 & 4.2231 & 4.0000 & 1.6786 & 6.0000 & 4.6428 & 8.0000 & 4.5117 \\
\hline Swr158 & 18.0000 & 7.8165 & 8.0000 & 4.5012 & 9.0000 & 5.3985 & 13.0000 & 7.9421 \\
\hline Sw951 & 14.0000 & 7.4851 & 7.0000 & 4.3484 & 9.0000 & 6.6736 & 11.0000 & 6.0063 \\
\hline Sw271 & 13.0000 & 7.6035 & 7.0000 & 3.5527 & 7.0000 & 5.3838 & 6.0000 & 4.4802 \\
\hline S0386 & 11.0000 & 1.6937 & 4.0000 & 1.1791 & 5.0000 & 1.6575 & 5.0000 & 2.3995 \\
\hline S0068 & 10.0000 & 4.3803 & 4.0000 & 2.1718 & 3.0000 & 2.9798 & 10.0000 & 2.6957 \\
\hline Swr1008 & 10.0000 & 2.9943 & 8.0000 & 2.3496 & 3.0000 & 1.4226 & 6.0000 & 3.1874 \\
\hline S0007 & 15.0000 & 8.3485 & 7.0000 & 2.8348 & 6.0000 & 3.6264 & 11.0000 & 6.6276 \\
\hline Sw857 & 15.0000 & 6.6685 & 8.0000 & 4.7574 & 6.0000 & 2.8017 & 12.0000 & 7.6270 \\
\hline Sw886 & 12.0000 & 7.7639 & 6.0000 & 3.6060 & 6.0000 & 4.8583 & 8.0000 & 5.4294 \\
\hline Sw919 & 9.0000 & 5.7641 & 6.0000 & 2.8058 & 3.0000 & 2.3525 & 4.0000 & 2.5938 \\
\hline Swr312 & 11.0000 & 5.6995 & 7.0000 & 4.9536 & 6.0000 & 3.7909 & 8.0000 & 4.4802 \\
\hline Sw81 & 8.0000 & 5.6566 & 6.0000 & 2.3297 & 3.0000 & 1.9552 & 7.0000 & 3.6961 \\
\hline Swr1120 & 11.0000 & 6.7385 & 8.0000 & 2.6224 & 5.0000 & 2.9077 & 7.0000 & 4.0896 \\
\hline Sw787 & 9.0000 & 5.1087 & 6.0000 & 2.4961 & 4.0000 & 3.8672 & 6.0000 & 4.7574 \\
\hline S0062 & 12.0000 & 7.1068 & 6.0000 & 2.4961 & 5.0000 & 4.0549 & 10.0000 & 6.0631 \\
\hline S0218 & 11.0000 & 6.1515 & 6.0000 & 1.5741 & 6.0000 & 3.6679 & 5.0000 & 4.1068 \\
\hline MV & 12.125 & 5.8748 & 5.7500 & 2.5727 & 6.1875 & 3.7837 & 8.1250 & 4.6470 \\
\hline
\end{tabular}

OAN means observed allele number. EAN means effective allele number. MV means mean value.

Table 4. Genetic structure indexes of the three miniature stocks through analyzing the PCR products of the 32 microsatellites combination.

\begin{tabular}{lcccc}
\hline Breed & Mean number of alleles & Mean effective number of alleles & Mean heterozygosity & PIC \\
\hline BM & 5.7500 & 2.5727 & 0.5428 & 0.5269 \\
GZ & 6.1875 & 3.7837 & 0.6978 & 0.6796 \\
TB & 8.1250 & 4.6470 & 0.7646 & 0.7463 \\
\hline
\end{tabular}

BM means Bama miniature pig. GZ means Guizhou miniature pig. TB means ongoing breeding Tibeta miniature pig. PIC means polymorphism information content.

Table 5. Nei's genetic distance (above diagonal) and Nei's standard genetic distance (below diagonal) among the three miniature swine outbred stocks.

\begin{tabular}{lccc}
\hline Population & BM & GZ & TB \\
\hline BM & - & 0.4207 & 0.3199 \\
GZ & 0.8659 & - & 0.3943 \\
TB & 1.1397 & 0.9307 & - \\
\hline
\end{tabular}

BM means Bama miniature pig. GZ means Guizhou miniature pig. TB means ongoing breeding Tibeta miniature pig. 


Table 6. Genetic structure indexes of CJ-1 analyzed by the 32 microsatellite combination.
\begin{tabular}{lcccc}
\hline Breed & Mean number of alleles & Mean effective number of alleles & Mean heterozygosity & 0.6903 \\
\hline CJ-1 & 6.1613 & 3.8483 & PIC \\
\hline CJ-1 means Changehun-junmo-1 outbred pig stock. PIC means polymorphism information
\end{tabular}

\section{DISCUSSION}

In this study, 32 microsatellites distributed across almost all chromosomes were screened out to study the swine population genetic characters of the Chinese outbred stocks $\mathrm{BM}, \mathrm{GZ}, \mathrm{TB}$ and CJ-1. According to a previous study, the DNA markers used in the genetic research are more important than the sample number collected (Nei et al., 1983). Ideally, the more microsatellite markers used to analyze the population genetic structure, the more reliable data one can get. However, for economic and efficiency reasons, it is preferable to use as few DNA markers as possible. As a result, the microsatellite loci used for population genetic study should have the following minimum qualifications, such as employing at least 25 loci (four alleles for each locus) and having no linkage relations between the loci (Benavides et al., 2000). In this study, there were 7 additional markers to the required 25, and most of the loci had more than 4 effective allele numbers (except for S0386 and swr1008), which may represent a comprehensive microsatellite combination and will supply multiple choices for various research objectives.

To obtain representative loci with reliable polymorphisms in our research, 3 electrophoretic methods, including $1.5 \%$ agarose gel, $8 \%$ polyacrylamide gel and capillary electrophoresis, were employed for step by step selection according to their respective resolution peculiarities of nucleotides from 10 on agarose gel, 5 on polyacrylamide gel, and 1-2 in capillary electrophoresis. As has been noted, a higher mean effective number of alleles indicates that the population is stable enough to evade new variation when faced with the pressure of genetic drift and natural and artificial selections (Takezaki and Nei, 1996). The results of our selection showed that the mean effective allele number per locus among the 3 stocks was nearly 5.9. But the mean effective allele value in both BM (2.6) and GZ (3.8) were smaller than the recommended value (4 alleles for each locus), which might have been because both stocks had half-sibling mating histories before they were isolated from their original habitats, and because the general population of each stock was not large enough in the breed.

Our study used 3 methods to perform genetic monitoring and population study of pigs for different research purposes and for performing the assay under various laboratory qualifications on the basis of a 32-locus combination. On the one hand, from an economic and convenient perspective, agarose and polyacrylamide gel electrophoresis, due to their simple, rapid, economic and little equipment need, should be favorable methods. On the other hand, although the costs and technical requirements are disadvantages for capillary scanning, it provides precise results for special studies.

Population genetic statistics that were developed to infer the population structures of naturally occurring populations are commonly introduced to describe the genetic diversity and structure of livestock (Wilkinson et al., 2011). The present results were consistent with the outbred stock breeding pedigrees and recorded generations, and thus, our study indicated that the 32-locus combination markers could efficiently reflect the basic characters of each stock studied. First the mean heterozygosities of the three stocks were different from each 
other, varying from 0.5428 in the $\mathrm{BM}$ pig (more than 20 generations of breeding), 0.6903 in CJ-1 (more than 20 generations) and 0.6978 in GZ (more than 15 generations of breeding) to 0.7646 in TB pig (4 generations of breeding). This implied that the heterozygosity of a breed was strictly related to the generations bred. Second, the three stocks showed different PIC values, implying that the microsatellite locus polymorphisms within each stock were very evident. The degree of hereditary variation of the 3 miniature swine from low to high was $\mathrm{BM}<\mathrm{GZ}<\mathrm{CJ}-1<\mathrm{TB}$. Generally, the lower the heterozygosity of a population, the smaller the genetic variation is in the population. Accordingly, both BM and GZ pigs had more stable genetics if compared to the standard outbred stock of CJ-1, whose mean heterozygosity was 0.6903. Notably, BM pig was more like a well-established outbred miniature swine stock than the other two, whereas TB was far from being a standard outbred stock so far.

We noticed that the mean heterozygosity and PIC of GZ and BM outbred pigs shown in this study were different from previous reports. For instance, in GZ stock, the reported mean heterozygosity value was 0.5562 (Huang et al., 2000), 0.67-0.83 (Yao et al., 2006) or 0.6602 (Wang et al., 2002); in BM, the value was 0.5990 (Wang et al., 2008). The PIC values in our study were much higher than in former reports. For example, the value for GZ was 0.4253 , while 0.3520 for BM (Shang et al., 2001). Although the microsatellite loci used by these studies were derived from either Roher et al. (1994) or recommended by FAO, it was hard to compare these reports to ours because they either used fewer DNA markers (10 loci) (Wang et al., 2002), or used fewer samples (10 pigs) and/or markers (nine loci) (Huang et al., 2000; Shang et al., 2001), or because the samples' origin differed compared to ours (Yao et al., 2006).

Fortunately, our results basically matched the breeding pedigrees and recorded generations of the breeds of the 3 miniature stocks and CJ-1, and the sampling number for each stock was relatively large (more than 35/stock). The results indicated that the genetic study employing the 32-locus combination selected was reliable and applicable, and that the combination could be the representative DNA markers for genetic monitoring and population structure analysis.

China has more pig breeds than any other country in the world so far, most of which are still local and show a large genetic difference between each other (Li et al., 2004; Megens et al., 2008). According to this study, the genetic distance between TB and BM was further than from GZ, but BM and GZ had a closer relation to each other. The reason may be that geological distance between BM and GZ is closer, whereas TB is a special kind of a few breeds that adapt to harsh environmental conditions. Our results were consistent with Shang et al. (2001).

Although pooling DNA samples during PCR procedures may lead to a bias in the allele frequency, it provides a cost-effective method for any study related to microsatellite markers (Megens et al., 2008). In agreement with this viewpoint, 9 DNA samples that were evenly coming from the three Chinese miniature swine outbred stocks were mixed together before they were used as the DNA samples. Possibly, this may help to minimize the variations that could exist between individual pigs, and to amplify as many polymorphic alleles as possible for each microsatellite locus.

Taken together, the selected 32-locus combination provides an alternative choice for genetic monitoring of the quality and the population genetic structure of outbred stocks.

\section{ACKNOWLEDGMENTS}

Research supported by the National Science Foundation of China (\#31172168 and \#31272393), the Key Projects in the National Science \& Technology Pillar Program 
(\#2011BAI15B01-32) and the Beijing Natural Science Foundation (\#5122005). We thank Guangxi University, Guizhou College of Traditional Chinese Medicine, Southern Medical University \& Shenzhen Bright Farm Group, and Ji Lin University for kindly providing samples of miniature swine and CJ-1.

\section{Supplementary material}

\section{REFERENCES}

Benavides F, Stern MC, Glasscock E, DiGiovanni J, et al. (2000). Microsatellite DNA variants between the inbred SENCAR mouse strains. Mol. Carcinog. 28: 191-195.

Bode G, Clausing P, Gervais F, Loegsted J, et al. (2010). The utility of the minipig as an animal model in regulatory toxicology. J. Pharmacol. Toxicol. Methods 62: 196-220.

Cao HC, Yang JF, Yu J, Pan QL, et al. (2012). Therapeutic potential of transplanted placental mesenchymal stem cells in treating Chinese miniature pigs with acute liver failure. BMC Med. 10: 56.

Chang WH, Chu HP, Jiang YN, Li SH, et al. (2009). Genetic variation and phylogenetics of Lanyu and exotic pig breeds in Taiwan analyzed by nineteen microsatellite markers. J. Anim. Sci. 87: 1-8.

Droc G, Grigorescu B, Grigoroiu M, Tomulescu V, et al. (2009). Anesthesia for experimental surgery in swine. Chirurgia 104: 259-265.

Esteban MA, Xu JY, Yang JY, Peng MX, et al. (2009). Generation of induced pluripotent stem cell lines from Tibetan miniature pig. J. Biol. Chem. 284: 17634-17640.

Fang M, Hu X, Jin W, Li N, et al. (2009). Genetic uniqueness of Chinese village pig populations inferred from microsatellite markers. J. Anim. Sci. 87: 3445-3450.

Fredholm M, Wintero AK, Christensen K, Kristensen B, et al. (1993). Characterization of 24 porcine (dA-dC)n-(dT-dG)n microsatellites: genotyping of unrelated animals from four breeds and linkage studies. Mamm. Genome 4: 187-192.

Huang ZB, Niu R, Wei H and Gan SX (2000). Monitoring the genetic variation of Guizhou miniature pigs with STR-PCR. Acta Acad. Med. Militaris Tertiae 22: 642-644.

Kim TH, Kim KS, Choi BH, Yoon DH, et al. (2005). Genetic structure of pig breeds from Korea and China using microsatellite loci analysis. J. Anim. Sci. 83: 2255-2263.

Li SJ, Yang SH, Zhao SH, Fan B, et al. (2004). Genetic diversity analyses of 10 indigenous Chinese pig populations based on 20 microsatellites. J. Anim. Sci. 82: 368-374.

May B, Krueger CC, Eng C and Paul E (1995). Genes in Populations, Version 2.0: A Computer Program for Analysis of Genetic Data. Cornell Laboratory for Ecological and Evolutionary Genetics, Cornell University, Ithaca.

Megens HJ, Crooijmans RPMA, Cristoba MS, Li XN, et al. (2008). Biodiversity of pig breeds from china and Europe estimated from pooled DNA samples: differences in microsatellite variation between two areas of domestication. Genet. Sel. Evol. 40: 103-128.

Mortensen JT, Brinck P and Lichtenberg J (1998). The minipig in dermal toxicology. A literature review. Scand. J. Lab. Anim. Sci. 25: 77-83.

Nei M (1995). Genetic distances between populations. Anim. Naturelist. 106: 283-292.

Nei M, Tajima F and Yateno Y (1983). Accuracy of estimated phylogenetic trees from molecular data. II. Gene frequency data. J. Mol. Evol. 19: 150-173.

Rohrer GA, Alexander LJ, Keele JW, Smith TP, et al. (1994). A microsatellite linkage map of the porcine genome. Genetics 136: 231-245.

Ross JW, Juan P, Fernandez DC, Zhao JG, et al. (2012). Generation of an inbred miniature pig model of retinitis pigmentosa. Invest. Ophth. Vis. Sci. 53: 501-507.

Shang HT, Niu R, Wei H, Huang ZB, et al. (2001). Genetic analysis of 35 microsatellite loci in three miniature pig breeds. Heriditas 23: 17-20.

Sneath PHA and Sokal RR (1973). Numerical Taxonomy. W.H. Freeman, San Francisco.

Sun BX, Hou WW, Xu ZS, Xie ZC, et al. (2000). Selection index combined with stress-sensitive genotype in swine breeding. Swine Prod. 1: 31-32.

Sun Y, Jiang Q, Tian DY, Lin H, et al. (2011). Experimental infection of Bama miniature pigs with a highly virulent classical swine fever virus. Virol. J. 8: 452.

Takezaki N and Nei M (1996). Genetic distances and reconstruction of phylogenetic trees from microsatellite DNA. Genetics 44: 389-399. 
Wang X, Cao HH, Gen SM, Li HB, et al. (2002). Chinese miniature pigs' genetic diversity by using microsatellite marker. Acta Vet. Zootech. Sin. 33:530-532.

Wilkinson S, Haley C, Alderson L and Wiener P (2011). An empirical assessment of individual-based population genetic statistical techniques: application to British pig breeds. Heredity 106: 261-269.

Yang SL, Wang ZG, Liu B, Zhang GX, et al. (2003). Genetic variation and relationships of eighteen Chinese indigenous pig breeds. Genet. Sel. Evol. 35: 657-671.

Yao SK, Zhang Q, Sun FZ and Liu PQ (2006). Genetic diversity of seven miniature pig breeds (strains) analyzed by using microsatellite markers. Hereditas 28: 407-412.

Zuo B, Du X, Zhao J, Yang H, et al. (2012). Analysis of microsatellite polymorphism in inbred knockout mice. PloS One e34555. 